\title{
C-C Bond Formation with Boronic Acids and Derivatives by Transition-Metal-Free Conjugate Addition Reactions
}

\section{Francisco Sánchez-Sancho}

Aurelio G. Csákÿ*

Synthesis 2016, 48, 2165.

For the final online and print versions, the title and reference 14 have been corrected. 